\title{
Paraneoplastic hypoglycaemia secondary to IGF-2 secretion from a metastatic gastrointestinal stromal tumour
}

\author{
Michael C. Onyema ${ }^{a}$, Eftychia E. Drakou ${ }^{b}$, Georgios Giovos ${ }^{c}$, Bianca M. Leca ${ }^{c}$, \\ Christos Kosmas $^{\mathrm{d}}$, Ratnadeep Ganguly ${ }^{\mathrm{e}}$, Narasimha Murthy ${ }^{\mathrm{c}}$, Ashley B. Grossman ${ }^{\mathrm{f}, \mathrm{g}, \mathrm{h}}$, \\ Harpal S. Randeva ${ }^{c, i, j}$, Georgios K. Dimitriadis ${ }^{a, c, i, *}$ \\ a Department of Endocrinology and Metabolism, King's College Hospital NHS Foundation Trust, Denmark Hill, London, SE5 9RS, UK \\ b Department of Clinical Oncology, Guy's Cancer Centre - Guy's and St Thomas' NHS Foundation Trust, Great Maze Pond, London, SE1 9RT, UK \\ ${ }^{\mathrm{c}}$ Human Metabolism Research Unit, WISDEM Centre, University Hospitals Coventry and Warwickshire NHS Trust, Coventry, CV2 2DX, UK \\ ${ }^{\mathrm{d}}$ Hematopoietic Cell Transplant Unit, Division of Medical Oncology, Department of Medicine, "Metaxa" Cancer Hospital, Piraeus, Greece \\ e Pathology Labs, UHCW NHS Trust, Coventry, CV2 2DX, UK \\ ${ }^{\mathrm{f}}$ Oxford Centre for Diabetes, Endocrinology and Metabolism, University of Oxford, Oxford, OX3 7LE, UK \\ ${ }^{g}$ Barts and the London School of Medicine, Centre for Endocrinology, William Harvey Institute, London, EC1M 6BQ, UK \\ ${ }^{\mathrm{h}}$ Neuroendocrine Tumour Unit, Royal Free Hospital, London NW3 2QG \\ ${ }^{i}$ Division of Translational and Experimental Medicine-Metabolic and Vascular Health, Warwick Medical School, University of Warwick, Coventry, CV4 7AL, UK \\ ${ }^{\mathrm{j}}$ Division of Life and Health Sciences, Aston University, Birmingham, B4 7ET, UK
}

\section{A R T I C L E I N F O}

\section{Keywords:}

Paraneoplastic

Hypoglycaemia

Non-islet cell tumour

GIST

Imatinib

IGF-2

\begin{abstract}
A B S T R A C T
We report the case of a 79-year-old male with previous history of non-Hodgkin's lymphoma in remission, who presented acutely to the Accident and Emergency department with recurrent episodes of hypoglycaemia. At the time of presentation, a random glucose was low at $1.4 \mathrm{mmol} / 1$, which upon correction resolved his symptoms. In hindsight, the patient recalled having had similar episodes periodically over the past 2 months to which he did not give much notice. While hospitalized, he continued having episodes of symptomatic hypoglycaemia, requiring treatment with intravenous dextrose and per os steroids. Once stable, he was discharged on oral prednisolone and dietary advice. A computed tomography scan performed during inpatient stay showed multiple deposits in the abdomen. An ultrasound guided biopsy of one of the liver deposits was performed. Immunohistochemistry supported the diagnosis of a gastrointestinal stromal tumour (GIST) positive for CD34 and CD117. The diagnosis of non-islet cell tumour hypoglycaemia (NICTH) secondary to an IGF2 secreting GIST was confirmed with further biochemical investigations (IGF2=105.9nmol/1; IGF2:IGF1 ratio 23, Upper Level of Normal (ULN) <10). Targeted cytoreductive treatment with Imatinib mesylate following assessment of the tumour's mutational status was successful in preventing hypoglycaemia over a 21-month follow-up observation period.
\end{abstract}

Introduction

Non-islet cell tumour hypoglycaemia (NICTH) is now thought to be most often due to over-secretion of insulin-like growth factor-2 (IGF2). IGF-2 precursor is secreted by such tumours and is less able to bind to circulating binding proteins, such that it can pass through capillaries and thus activate the insulin receptor. Malignant neoplasms of mesenchymal and epithelial origin may secrete IGF-2, causing in a minority of cases, fasting hypoglycaemia characteristic of insulin producing islet cells tumours (Dynkevich et al., 2013, Dimitriadis et al., 2017). Diagnosis is confirmed by the demonstration of hypoglycaemia in the presence of low levels of serum insulin and an elevated IGF-2/IGF-1 ratio, determined using peripheral blood samples. In a recent review by Han et al., 76 solid fibrous tumours presenting with NICTH were found in the literature. In 8 cases, there was over-secretion of growth hormone (GH) with only 1 case demonstrating decreased concentration. Furthermore, 4 cases reported presence of Insulin Growth Factor Binding Protein 3 (IGFBP3), in which 3 cases showed decreased levels, and1case did not have a reported value for the normal range of IGFBP3. In cases of manifested hypoglycaemia, C-peptide and insulin concentrations were marginally decreased. In 15 out of 19 cases, the IGF-2/IGF-1 ratio was $>10$. In addition, 4 cases reported IGF-2/IGF-1 ratio $<10$, while total IGF-2 concentrations were normal and showed significantly suppressed levels of IGF-1 (Han et al., 2017).

\footnotetext{
* Corresponding author at: Department of Endocrinology, King’s College Hospital NHS Foundation Trust, Denmark Hill, London, SE5 9RS, UK.

E-mail address: g.dimitriadis@warwick.ac.uk (G.K. Dimitriadis).
} 

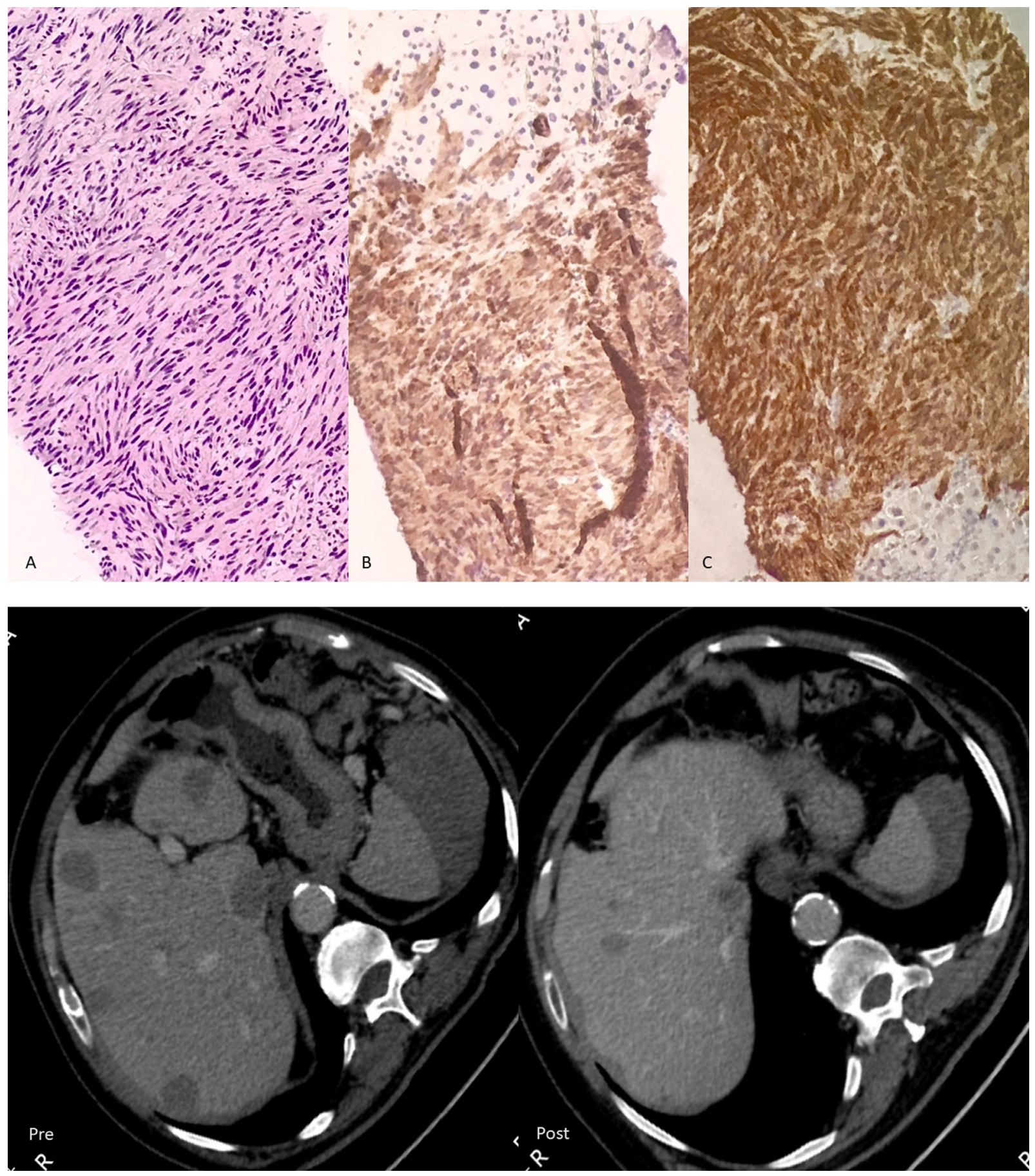

Fig. 1. A - Radiology legends: Pre. Contrast enhanced computerised tomography revealing extensive abdominal disease before treatment initiation with Imatinib mesylate. Post. Follow-up computerised tomography 6 months after treatment with Imatinib mesylate revealing partial disease response. B - Immunohistochemistry legends. A. Haematoxylin \& Eosin staining, 200X magnification light microscopy showing spindle cells arranged in fascicles and fibrillary eosinophilic cytoplasm forming whorls consistent with gastrointestinal stromal tumour (GIST). B\&C. Immunohistochemical studies showing tumour cells diffusely positive for proto-oncogene c-kit (CD 117) and DOG-1, magnification 200X.

The main treatment for NICTH depends on disease stage at presentation and this can vary between surgical excision or selective chemotherapy agents targeting $C$-kit pathogenic variants, with glucocorticoids and other treatments such as growth hormone used for symptomatic relief. (Indio 2018). Historically GIST has been considered radiation-resistant, and the role of radiotherapy in the management of patients with GIST is currently restricted to symptomatic palliation in current treatment guidelines (Gatto et al., 2017).

\section{Case report}

A 79-year-old Caucasian man, with history of follicular grade 2 non-Hodgkin's lymphoma in remission for more than 5 years following cytoreductive treatment, presented to the emergency department with weight loss, reduced appetite and recurrent episodes of hypoglycaemia for 2 months preceding admission. At the time of presentation, his $\mathrm{HbA} 1 \mathrm{c}$ was $34 \mathrm{mmol} / \mathrm{mol}$ (reference range $29-41 \mathrm{mmol} / \mathrm{mol}$ ) and 
blood glucose $1.4 \mathrm{mmol} / 1$ (reference range $4.0-5.6 \mathrm{mmol} / \mathrm{L}$ ) in the absence of glucose-lowering treatment. He was treated for hypoglycaemia using a local protocol including administration of intramuscular $1 \mathrm{mg}$ Glucagon and, once stable with oral dextrose tablets. During his stay, a short Synacthen test using $250 \mathrm{mcg}$ of intravenously administered tetracosactide was carried out to assess his adrenal reserves. His 60-minute cortisol rose above 550nmol/L excluding adrenal insufficiency according to Generation II Roche cortisol assay standards used by local pathology labs.

He was discharged with dietary advice and prednisolone $20 \mathrm{mg}$ once daily for 4 weeks to avoid recurrent hypoglycaemia with clear instructions to reduce the dose gradually until discontinued and he was reviewed again in the outpatient department. A contrast-enhanced CT scan of his abdomen did not demonstrate any pancreatic neoplasms; however revealed liver and omental deposits (Fig. 1A). A guided biopsy performed at a later stage with subsequent immunohistochemistry was positive for CD117 (C-kit), Dog1, CD34 with focal expression of SMA, confirming the diagnosis of a metastatic gastrointestinal stromal tumour (GIST) (Fig. 1B; A-C). Genetic testing for c-kit activating pathogenic variants following DNA isolation from paraffin-embedded tissue samples was carried-out to predict his response to therapy and guide the anti-CD117 starting dose with imatinib mesylate; this revealed a exon 11 deletion (Indio 2018). NICTH was suspected, with an IGF-2 (105.9 $\mathrm{nmol} / \mathrm{l})$ to IGF-1 $(4.6 \mathrm{nmol} / \mathrm{l})$ ratio of 23 supporting the diagnosis (UNL $<10$ ). Following discussion at the upper gastrointestinal malignancy multidisciplinary meeting, treatment with imatinib mesylate $400 \mathrm{mg}$ daily was initiated and prednisolone was gradually tapered off. Two months following initiation of treatment, there were no further reported hypoglycaemic episodes. Surveillance imaging six months after treatment initiation revealed a partial response (Fig. 1A) and there were no hypoglycaemic episodes for the next 21 months.

\section{Discussion}

The incidence of NICTH ranges annually from 2.1 to 17 per million people with even distribution between genders and is typically seen with large metastatic or mesenchymal tumours (Dimitriadis et al., 2017). NICTH presents as recurrent or persisting hypoglycaemic episodes and mostly affects elderly patients with advanced tumours. Occasionally, these hypoglycaemic episodes can predate the diagnosis of the underlying tumour, being the presenting symptom that leads to diagnosis of an underlying malignancy. NICTH is commonly associated with tumour cell production of IGF2. NICTH is characterised by low serum insulin and C-peptide levels, low growth hormone (GH) and IGF1 levels, normal or elevated IGF2 levels, and an elevated IGF2:IGF1 ratio. When available to measure, expression of 'big' IGF2 has been reported and rarely there is expression of pro-IGF2. High concentrations of pre-pro-IGF2 levels that are not properly glycosylated result in the high molecular weight 'big' IGF2 that has a significantly higher affinity to the insulin receptor, and a lower affinity to its binding protein (IGFBP3), leading to in- creased bioavailability, enhanced peripheral glucose consumption and suppressed hepatic glucose production (Dimitriadis et al., 2017).

The diagnosis of GIST is confirmed by immunohistochemical staining of CD117 (C-kit), which is positive in almost 95\% of cases (Dimitriadis et al., 2015, Garla et al., 2019). Pathogenic variant analysis in GIST is crucial as it identifies less sensitive or resistant genotypes (PDGFR $D 842 \mathrm{~V}$ ) to selective Tyrosine Kinase Inhibitor (TKI) imatinib mesylate and allows dose adjustment for patients with c-kit exon 9 pathogenic variants (800mg daily). This contrasts with the standard dose of the most common c-kit exon 11 pathogenic variant which is $400 \mathrm{mg}$ daily (Garla et al., 2019). Long-term use of glucocorticoids may be appropriate for the management of NICTH secondary to other mesenchymal neoplasms as these can suppress tumor-specific secretion of "big" IGF-II (Han et al., 2017). Surgical removal of the primary tumour remains the most rapid and effective therapy for normalising glucose metabolism in most cases of NICTH when indicated.

NICTH is a poorly recognised cause of hypoglycaemia and consequently may frequently remain undiagnosed. This case highlights the importance of recognising NICTH as a potential cause of emergency department attendance in cases of patients with advanced malignancy and adds to cases confirming GIST as the source of abnormal secretion of IGF-2.

\section{Supplementary materials}

The Peer Review Overview associated with this article can be found, in the online version, at doi:10.1016/j.endmts.2020.100047.

\section{References}

Dynkevich, Y., Rother, K.I., Whitford, I., Qureshi, S., Galiveeti, S., Szulc, A.L., Danoff, A., Breen, T.L., Kaviani, N., Shanik, M.H., Leroith, D., Vigneri, R., Koch, C.A., Roth, J., 2013. Tumors, IGF-2, and hypoglycemia: insights from the clinic, the laboratory, and the historical archive. Endocr Rev 34 (6), 798-826 Dec.

Dimitriadis, G.K., Angelousi, A., Weickert, M.O., Randeva, H.S., Kaltsas, G., Grossman, A., 2017. Paraneoplastic endocrine syndromes. Endocr Relat Cancer 24 (6), R173-R190 Jun.

Han, Guiyan, Zhang, Zhimin, Shen, Xingbin, Wang, Kunpeng, Zhao, Yang, He, Jianqiu, Gao, Yu, Shan, Xiujie, Xin, Guohua, Li, Chunhui, Liu, Xiaoyan, 2017. Doege-Potter syndrome - A review of the literature including a new case report. Medicine 96 (27), e7417.

Indio, 2018. Integrated Molecular Characterization of Gastrointestinal Stromal Tumors (GIST) Harboring the Rare D842V Mutation in PDGFRA Gene. Int J Mol Sci. 19 (3), 732 Mar.

Gatto, L., Nannini, M., Saponara, M., Di Scioscio, V., Beltramo, G., Frezza, G.P., Ercolani, G., Pinna, A.D., Astolfi, A., Urbini, M., Brandi, G., Biasco, G., Pantaleo, M.A., 2017. Radiotherapy in the management of gist: state of the art and new potential scenarios. Clin Sarcoma Res 7, 1 Jan 10.

Dimitriadis, G.K., Gopalakrishnan, K., Rao, R., Grammatopoulos, D.K., Randeva, H.S., Weickert, M.O., Murthy, N., 2015. Severe paraneoplastic hypoglycemia secondary to a gastrointestinal stromal tumour masquerading as a stroke. Endocrinology, Diabetes \& Metabolism Case Reports 1. doi:10.1530/EDM-15-0062.

Garla, V., Sonani, H., Palabindala, V., Gomez-Sanchez, C., Subauste, J., Lien, L.F., 2019. Non-islet Cell Hypoglycemia: Case Series and Review of the Literature. Front. Endocrinol. 10, 316. doi:10.3389/fendo.2019.00316. 